6 Black, H. S., and Rauschkolb, E. W., J. Invest. Dermatol., 56, 387 (1971).

7 Bischoff, F., in Advances in Lipid Research (edit. by Paoletti, R., and Kritchevsky, D.), 7, 165 (Academic Press, New York, 1969).

\section{Visual-Auditory Distance Constancy}

WE wish to describe a new perceptual constancy phenomenon which may be termed auditory distance constancy. If an observer views an event which produces a sound it seems that some compensation is made for the fact that the event will stimulate the eye somewhat before it will stimulate the ear depending on the distance of the event from the observer. For example, the sound of an event $35 \mathrm{~m}$ away will arrive at the ear about $100 \mathrm{~ms}$ after the light arrives at the eye.

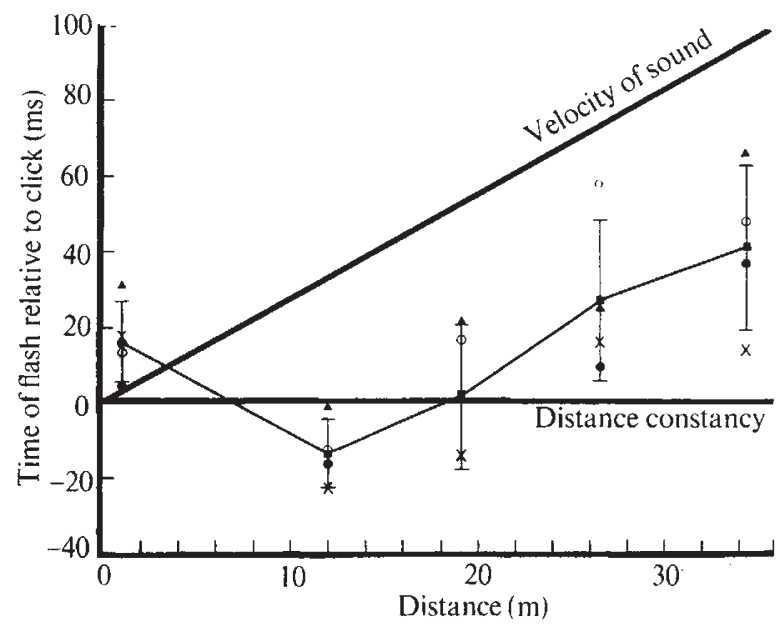

Fig. 1 Graph of the time delay of the flash relative to the click judged to represent a common event as a function of distance. Positive values represent the flash occurring after the click. $\times, S_{1} ; \bigcirc, S_{2} ; \boldsymbol{\Lambda}, S_{3} ; \bigcirc, S_{4} ; \mathbf{E}$, mean.

To investigate whether compensation is made for differences in arrival time of light and sound we asked observers to judge whether a light flash and a click were simultaneous as a function of distance. The light was a model PS2E Grass photo stimulator mounted in a box whose front was $47 \mathrm{~cm}$ wide and $36 \mathrm{~cm}$ high. As seen by the observer, the front of the box contained a white square $(15 \times 5 \mathrm{~cm})$ on a black background. The photo stimulator produced a brief illumination of the white square. The light box was set on a laboratory stool which was partly covered with a black cloth used to conceal a small loudspeaker. A $0.5 \mathrm{~ms}$ pulse fed to the speaker produced the click. Timing of the flash and the click was the function of two American Electronics Laboratories stimulators.

For the experiment an observer was seated at one end of a long corridor and the light box and speaker were located at various distances down the corridor. The corridor gave good distance cues. The light flash and click were presented with various delays between the flash and the click. The delays of the flash relative to the click were $-200,-150,-100,-50$, $0,50,100,150$ and $200 \mathrm{~ms}$. (A positive delay means the click occurred before the flash.) The observer was asked to adopt the attitude that the click and the flash would ordinarily be the same event. Thus for a given click -flash combination he had to decide whether the combination met the criterion of representing a single event. The distances used in the experi- ment were $1,11.9,19.2,26.5$ and $34.4 \mathrm{~m}$. Four observers were used in the experiment. For two observers each clickflash delay was presented ten times at each distance. For the other two observers each combination was presented fifteen times.

The results of the experiment are shown in the graph of Fig. 1. The ordinate represents the average delay of the flash relative to the click judged by each observer to represent a common event at each distance. The abscissa represents the distance at which the observation was made. A line is drawn through the mean delay for all four observers. Also shown is the standard deviation of the observers.

In addition to the observed data two theoretical lines are shown in Fig. 1. The horizontal line through zero delay represents the observations that would be obtained if the observers took perfect account of distance and the velocity of sound. The increasing straight line function represents observations that would be obtained if the observer took no account of distance and velocity but based their judgments on the simultaneous arrival of the sound and light at the observer. For this line the velocity of sound was taken as $344.45 \mathrm{~m} \mathrm{~s}^{-1}$ (calculated for dry air at $22^{\circ} \mathrm{C}$ ). By comparing the observations with the two theoretical lines it may be seen that the observers corrected for the velocity of sound for distances up to about $20 \mathrm{~m}$ beyond which the constancy begins to break down. Even at $35 \mathrm{~m}$, however, there is still compensation by a factor of 0.5 . As to the pattern of observations at the shorter distances it is difficult to say whether the apparent undercompensation at $1 \mathrm{~m}$ and overcompensation at $11.9 \mathrm{~m}$ is genuine or due to peculiarities of the situation.

A further observation worth noting on the graph is that the standard deviations of the observers' means at various distances are fairly small ranging from 9 to $22 \mathrm{~ms}$. On the other hand, the standard deviation for individual observers at each point ranged from 50 to $100 \mathrm{~ms}$. Thus the variability of the observers relative to one another was about half that which would be expected on the basis of the individual variability. Although judgments of perceived simultaneity are typically in the region of $50-100 \mathrm{~ms}$, this would not explain the small between-observer variability. We believe that the reason for the discrepancy between individual and group variability is that the distribution of judgments for an individual at a given distance may be bimodal, one mode corresponding to simultaneity of the flash and click at the observer and one to simultaneity at the source. To establish this, however, would require more careful measurements and longer distances.

Because the experimental results implied that observers compensated only partially for longer distances, a size constancy experiment was carried out to get estimates of the perceived distances. That is, the partial compensation could have resulted from underestimating the distances involved. The estimates were obtained by having each observer adjust a variable size white square on a black background, viewed at a standard distance of $3 \mathrm{~m}$, to match the absolute size of the white square in the light box as a function of the distance of the light box. The settings of the comparison square relative to the true size of the square on the light box were used to estimate the perceived distance of the light box. It was found that in general the perceived distances matched the true distances very well and that in no case would the observer's error in distance perception account for failure to compensate completely for the auditory time delay.

This work was supported by a National Research Council of Canada grant to G. R. E.

G. R. ENGEL

W. G. DOUGHerty

Department of Psychology,

University of Waterloo,

Waterloo, Ontario

Received May 28; revised June 25, 1971. 